\title{
Representation of Angles Embedded within Contour Stimuli in Area V2 of Macaque Monkeys
}

\author{
Minami Ito ${ }^{1}$ and Hidehiko Komatsu ${ }^{1,2}$ \\ ${ }^{1}$ Division of Sensory and Cognitive Information, Department of Information Physiology, National Institute for Physiological Sciences, Okazaki, Aichi 444- \\ 8585, Japan, and 2Department of Physiological Sciences, The Graduate University for Advanced Studies, Okazaki, 444-8585, Japan
}

\begin{abstract}
Angles and junctions embedded within contours are important features to represent the shape of objects. To study the neuronal basis to extract these features, we conducted extracellular recordings while two macaque monkeys performed a fixation task. Angle stimuli were the combination of two straight half-lines larger than the size of the classical receptive fields (CRFs). Each line was drawn from the center to outside the CRFs in 1 of 12 directions, so that the stimuli passed through the CRFs and formed angles at the center of the CRFs. Of 114 neurons recorded from the superficial layer of area V2, 91 neurons showed selective responses to these angle stimuli. Of these, 41 neurons $(36.0 \%)$ showed selective responses to wide angles between $60^{\circ}$ and $150^{\circ}$ that were distinct from responses to straight lines or sharp angles $\left(30^{\circ}\right)$. Responses were highly selective to a particular angle in approximately one-fourth of neurons. When we tested the selectivity of the same neurons to individual half-lines, the preferred direction was more or less consistent with one or two components of the optimal angle stimuli. These results suggest that the selectivity of the neurons depends on both the combination of two components and the responses to individual components. Angle-selective V2 neurons are unlikely to be specific angle detectors, because the magnitude of their responses to the optimal angle was indistinguishable from that to the optimal half-lines. We suggest that the extraction of information of angles embedded within contour stimuli may start in area V2.
\end{abstract}

Key words: angle; contour; area V2; primate; visual cortex; corner

\section{Introduction}

Distortion and disruption of contours provide important information enabling representation of the shape of objects as well as the contour lines themselves. These features are given by angles and junctions embedded within contours, and their partial replacement has a destructive effect on the appearance of objects. Although psychophysical studies have suggested the existence of special mechanisms sensitive to angles at relatively early visual stages (Chen and Levi, 1996; Heeley and Buchanan-Smith, 1996; Regan et al., 1996), the neuronal mechanisms responsible for extracting these features are not yet well understood. Our purpose in the present study was to clarify such neuronal mechanisms.

At higher visual stages, many neurons are selective for the complex shapes of objects (Gross, 1973; Desimone et al., 1984; Tanaka, 1993, 1996). In contrast, at area V1, the earliest visual stage, many neurons are highly selective for the orientation of line segments (Hubel and Wiesel, 1959, 1968; Hammond and Andrews, 1978). Their small classical receptive fields (CRFs) are quite appropriate for extracting local features of contour, such as

\footnotetext{
Received Sept. 25, 2003; revised Feb. 20, 2004; accepted Feb. 23, 2004.

This work was supported by the Japanese Grant-in-Aid for Scientific Research (C) 11680796 and (B) 14380371 We thank T. Ogawa for software development and M. Togawa and N. Takahashi for technical assistance.

Correspondence should be addressed to Minami Ito, Division of Sensory and Cognitive Information, Department of Information Physiology, National Institute for Physiological Sciences, Myoudaiji-cho 38, 0kazaki 444-8585, Japan. E-mail:minami@nips.ac.jp.

D0I:10.1523/JNEUROSCI.4364-03.2004

Copyright $\odot 2004$ Society for Neuroscience $\quad 0270-6474 / 04 / 243313-12 \$ 15.00 / 0$
}

fragments of short line segments. Thus, the neuronal mechanisms underlying object recognition might be a process of integrating this fragmentary information into a single object representation along the hierarchy of the visual pathways. At area V2, the next visual stage, many neurons are also selective for line segment orientation; moreover, Kobatake and Tanaka (1994) demonstrated that a few V2 neurons selectively respond to complex stimuli, including a sharp triangle, and suggested that the representation of complex stimulus features may begin to emerge in area V2. In that regard, Heider et al. (2000) showed that some single end-stop neurons showed selective responses to right corners, whereas Hegde and Van Essen (2000) showed that onethird of V2 neurons respond selectively to various complex stimuli, including angles. In these studies, however, only a small number of angle stimuli were used to evaluate response selectivity. Here, we systematically explored the response selectivity of V2 neurons in a large angle space.

Response selectivity has often been studied using small angle stimuli presented within CRFs as single complete objects (Kobatake and Tanaka, 1994; Hegde and Van Essen, 2000). Evidence suggests, however, that response selectivity is strongly influenced by contextual modulation from outside the CRF (for review, see Allman et al., 1985; Gilbert et al., 2000). In natural scenes, for instance, angles are presented as part of the contours of the objects and not as isolated visual stimuli. It therefore seems reasonable to take into account contextual modulation by contour lines to which angles are connected. We therefore prepared angle stimuli six to eight times larger than the size of the CRFs and report 
that many V2 neurons show angle selectivity that is dependent on the responses to the individual line components of the angle stimuli as well as to the combination of two line components. These results support the idea that signals encoding the orientation of individual line components converge in area V2, and this convergence is the first step to form the representation of angles within contour stimuli.

\section{Materials and Methods}

Two macaque monkeys (monkey CI: Macaca fuscata, female, weighing $3.9 \mathrm{~kg}$; monkey CM: Macaca mulatta, male, weighing $5.2 \mathrm{~kg}$ ) were trained to perform a fixation task and used for unit recordings. All procedures followed the National Institutes of Health Guide for the Care and Use of Laboratory Animals (revised 1996) and were approved by our institutional animal experimentation committee.

Training and preparation. We used three personal computers (PCs) to control the task sequence, to present visual stimuli, and to collect data. All program software was custom-made in our laboratory. Animals were first trained to sit in a monkey chair and perform a fixation task (rotation task; see below). They were then subjected to an initial surgical preparation: a stainless block for head fixation was attached to the top of the skull using cortical screws and dental cement, and scleral eye search coils (Judge et al., 1980) were implanted in both eyes to monitor eye movements (Enzanshi-Kogyo, Tokyo, Japan). All surgical procedures were performed under deep pentobarbital sodium anesthesia $(20 \mathrm{mg} / \mathrm{kg}$ supplemented with $10 \mathrm{mg} / \mathrm{kg}$ when necessary) and under aseptic conditions. After recovery, animals were provided with additional training until they stably performed the task, even when contour stimuli were presented in parafoveal visual fields as in the recording sessions.

Monkeys were required to fixate on a mark that appeared on the screen of a CRT display (Clinton Monoray Monitor, Cambridge Research Systems, Rochester, UK). We set three virtual fixation windows of different size around the fixation mark. When an animal's eye position moved within an inner fixation window $\left(0.5^{\circ} \times 0.5^{\circ}\right)$, recording trials began. The animal had to maintain fixation within an outer fixation window $\left(1.5^{\circ} \times 1.5^{\circ}\right)$ throughout the trial; otherwise the trial was aborted, and a large cross indicating an error was presented for $500 \mathrm{msec}$. When a monkey's eye movements were maintained within a middle fixation window $\left(1.0^{\circ} \times 1.0^{\circ}\right)$, physiological data were accepted for further analysis. During the fixation period, a stimulus was presented for a period of $200 \mathrm{msec}$, and then the presentation was repeated with an interstimulus interval of $900 \mathrm{msec}$. The repetition number varied randomly from one to three. After the error trials, the repetition number was fixed at one until the animals responded correctly. This served to encourage the animals to continue the task by shortening the total fixation period and making the task easier. After a post-stimulus period varying from 200 to $1200 \mathrm{msec}$, a vertical dark line on the fixation mark rotated by $90^{\circ}$, and monkeys had to press a lever within a short interval (300 and $500 \mathrm{msec}$ for each animal). The intertrial interval was $1 \mathrm{sec}$. Training or recording sessions were conducted $5 \mathrm{~d}$ /week, during which time water intake was restricted in the cage. Animals were given liquid reward for correctly performing the fixation task and were returned to their cages when they stopped performing the task. We monitored the animals' daily food intake and body weight and provided supplemental water if necessary.

Electrophysiological recordings. A metal recording chamber with an inner diameter of $22 \mathrm{~mm}$ was implanted over the left hemisphere in a position to allow access to areas V1 and V2 on the cortical surface. After training on the fixation task was completed, trephination was performed inside the chamber. Penetrations were then made through the dura matter using glass-coated platinum iridium microelectrodes (Wolbarsht et al., 1960) with impedances between 1.0 and $3.0 \mathrm{M} \Omega$ at $1 \mathrm{kHz}$. The electrodes were driven using a stepping motor microdriver (MO-95, Narishige, Tokyo), and successive penetrations were usually positioned $0.5 \mathrm{~mm}$ apart. Neuronal signals were amplified $(10,000 \times, 300 \mathrm{~Hz}-5 \mathrm{kHz}$; Model 1800, A-M Systems, Sequim, WA) and fed to a PC at a sampling rate of 25 $\mathrm{kHz}$ (analog-to-digital converter; PCI-416, Datel, Mansfield, MA). We used spike sorting to isolate single unit recordings during off-line analysis. The recording chamber was filled with agarose during recording
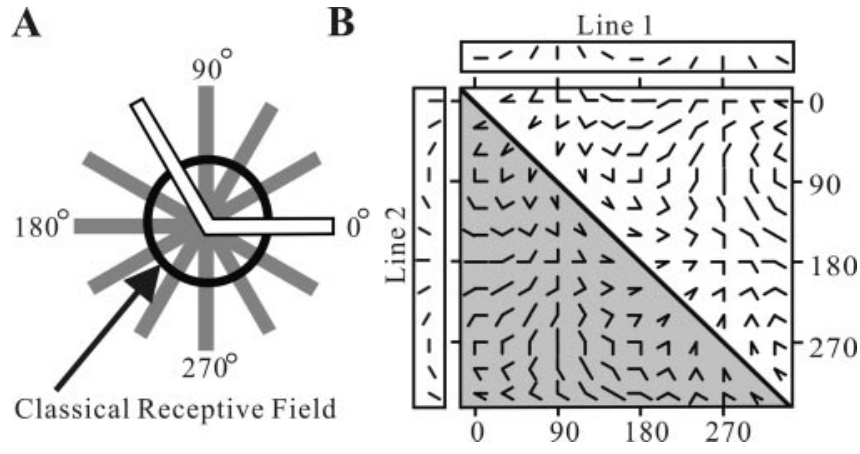

Figure 1. A, Angle stimuli were composed of combinations of two long half-lines. An example of an angle stimulus composed of $0^{\circ}$ and $120^{\circ}$ half-lines is indicated by white lines. Each half-line was drawn from the center to the outside of the CRF of the recorded neuron, so that stimuli passed through the CRF and formed an angle at its center. $B$, Angle space representing the entire set of angle stimuli used in this study. A set of 66 angle stimuli was made by changing the orientation of the half-lines in $30^{\circ}$ steps (illustrated at the top and left) and presented as a $12 \times 12$ matrix. The two half-lines were exchangeable, and the gray region of the matrix is a mirror image of the white region.

sessions to prevent the dura surface from drying and to reduce movement of cortical surfaces. Once the neuronal activity of either a single unit or a cluster of a few units was isolated, we determined the optimal orientation and optimal size of a bar stimulus by moving it manually and listening to the evoked discharges on an audio monitor. We determined the location and extent of the CRF for each recorded unit using the minimum response technique with an optimal bar stimulus (Barlow et al., 1967). We then began quantitative receptive field mapping and exploration of the response properties to angles.

After each recording session, the electrode and microdrive were removed, and the chamber was disinfected with Hibiten gluconate solution ( $0.05 \%$ chlorhexidine gluconate, Sumitomo Pharmaceuticals, Osaka, Japan) and filled with antibiotic (chloramphenicol-fradiomycin sulfateprednisolone ointment, Sankyo, Tokyo, Japan) before being sealed. Connective tissue on the dura matter was periodically stripped under anesthesia (ketamine hydrochloride, $10 \mathrm{mg} / \mathrm{kg}$, i.m.) to facilitate electrode penetration.

Visual stimuli. Visual stimuli were generated using a PC with a VSG2/3 graphics board (Cambridge Research Systems, Rochester, UK) controlled by custom software and displayed on a CRT display (Multisync Clinton Monoray; resolution, $800 \times 600$ pixels; refreshment rate, $60 \mathrm{~Hz}$; Cambridge Research Systems). The CRT was placed $110 \mathrm{~cm}$ in front of the animals, and the display covered a visual angle of $16^{\circ} \times 12^{\circ}$. Stationary stimuli were presented against a background of $4.0 \mathrm{~cd} / \mathrm{m}^{2}$ luminance. In most cases, bright contour stimuli (bars and angles of $0.06-0.10^{\circ}$ width) were presented at high contrast $\left(22.4 \mathrm{~cd} / \mathrm{m}^{2}\right)$; otherwise, dark stimuli were presented at high contrast $\left(0.5 \mathrm{~cd} / \mathrm{m}^{2}\right)$. For quantitative CRF mapping, a short line segment $\left(0.5-1.0^{\circ}\right.$ in length) of optimal orientation was presented within a $5 \times 5$ matrix with $0.5^{\circ}$ intervals. To study the properties of the responses to angle stimuli, we prepared a standard stimulus set by combining two straight "half-lines" $3^{\circ}$ or $4^{\circ}$ in length (Fig. $1 \mathrm{~A})$. Each half-line was drawn from the center to outside the CRF in 1 of 12 directions (every $30^{\circ}$ ), so that the stimulus passed through the CRF and formed an angle at the center. The example in Figure $1 A$ shows an angle stimulus composed of $0^{\circ}$ and $120^{\circ}$ half-lines. We made an angle space of all 66 combinations of two half-lines, which is shown as a $12 \times 12$ matrix in Figure $1 B$. The bottom left half of the matrix (gray region) is a mirror image of the top right half (white region). We did not include single half-lines in the above set of angles, although they may be regarded as a special case in which the two half-lines overlap. This was because the total length and brightness of the single half-line are different from those of other angle stimuli. They are indicated by diagonal lines in the matrix and the response profiles (see below). As a part of the stimulus set, we also studied responses to individual long $\left(3^{\circ}\right.$ or $\left.4^{\circ}\right)$ and short $\left(0.3^{\circ}\right)$ half-lines oriented in 12 directions and to short line segments $\left(0.6^{\circ}\right)$ presented at the centers of the CRFs in six orientations. Long line segments $\left(6^{\circ}\right.$ or $\left.8^{\circ}\right)$ 
in six orientations were included in the stimulus set as angle stimuli of $180^{\circ}$ width. We refer to the response selectivity for these half-lines as "direction selectivity" to distinguish it from ordinary "orientation selectivity."

Data collection and analysis. Data were collected for 5-10 repetitions, with each stimulus presented in a pseudorandom block design. Each cycle of recording periods started $-200 \mathrm{msec}$ from onset of stimulus presentation and stopped $200 \mathrm{msec}$ after offset of stimulus presentation. Eye positions were fed to PCs at $1 \mathrm{kHz}$, enabling us to analyze the relationship between eye positions and neuronal responses on a trial-by-trial basis. Spike occurrence during prestimulus periods provided the background firing rate for each neuron. Mean firing rates during stimulus presentation were measured from a time window $200 \mathrm{msec}$ in width, taking into account the response latency, so that maximal rates were obtained. Response magnitude was given by the mean firing rate during stimulus presentation minus the mean firing rate of the background activity. The statistical significance of the response to each stimulus was evaluated using Kolmogorov-Smirnov tests; firing rates for one stimulus were compared with those for background activity. Values of $p<0.05$ were considered significant.

To assess the distribution of the response activities of each unit within the angle space of 66 angle stimuli, mean firing rates were normalized to the maximal response and then plotted in a format similar to the stimulus matrix, which we will refer to as the "response profile." To make response profiles, we assigned appropriate directions a value of $0^{\circ}$, so that a continuous distribution of response activities could be presented as a single area within the response profile.

To evaluate optimal angles, we smoothed the response profile using a $3 \times 3$ Gaussian filter with the following coefficients: $(0.063,0.125,0.063)$, $(0.125,0.250,0.125),(0.063,0.125,0.063)$. In this way we avoided picking up local peaks in the response profile. We defined a separate response area using the threshold of $80 \%$ of the maximal response and assigned peaks for each response area. Some neurons had a secondary response area, hence the secondary peak. It should be noted that the smoothed profiles were used only to obtain the optimal angles and that further analyses were done with the original response profiles.

To compare the maximal responses to angles and half-lines, an angle response index $\left(I_{\mathrm{A}}\right)$ was computed using the following equation: $I_{\mathrm{A}}=$ $\left(R_{\mathrm{MA}}-R_{\mathrm{MHL}}\right) /\left(R_{\mathrm{MA}}+R_{\mathrm{MHL}}\right)$, where $R_{\mathrm{MA}}$ is the maximal response to the angle set and $R_{\mathrm{MHL}}$ is the maximal response to the half-lines. A value of 0.33 (or -0.33 ) means the response to the angle stimuli is two (or one-half) times as large as that to the half-lines.

To evaluate the specificity of the response selectivity to angle stimuli, we defined the "peak response area" as the extent of the continuous area of the response profile within which the response amplitude was $\geq 50 \%$ of the maximum. To characterize the distribution of responses in the response profile, we calculated the extent of the elongation along four axes centered at the optimal angle. Each axis represents a feature common to response-inducing angle stimuli, including the line components and the width and orientation of the angles (see Fig. 6). To compare the extent of elongation along three axes-primary (or secondary) line component ("line"), angle width ("wid"), and angle orientation ("ori")—we plotted the barycenter $(\vec{R})$ of the three parameters in a triangular area (see Fig. 7), as given by the following equation:

$$
\overrightarrow{\mathrm{R}}=\left(\mathrm{S}_{\text {line }} \overrightarrow{\mathrm{R}}_{\text {line }}+\mathrm{S}_{\text {wid }} \overrightarrow{\mathrm{R}}_{\text {wid }}+\mathrm{S}_{\text {ori }} \overrightarrow{\mathrm{R}}_{\text {ori }}\right) /\left(\mathrm{S}_{\text {line }}+\mathrm{S}_{\text {wid }}+\mathrm{S}_{\text {ori }}\right),
$$

where $\overrightarrow{\mathrm{R}}_{\text {line }}, \overrightarrow{\mathrm{R}}_{\text {wid }}, \overrightarrow{\mathrm{R}}_{\text {ori }}$ are vector representations of three corners of a triangular area, and Sline, Swid, and Sori are numbers of responseinducing stimuli equal to or larger than half the maximal response along the three axes, scaled by the maximal range $(0.0-1.0)$.

Recording sites. We recorded from the opercular surface of the occipital cortex in the lower parafoveal region near the V1-V2 border. The border was determined from the retinotopical organization of CRF positions and the changes in CRF size when the recording tracks crossed the V1-V2 border. Mapping of the size and location of the CRFs assured that recordings were from area V2. Because both animals will be used for additional recordings, no histological confirmation was obtained; however, one monkey was scanned using magnetic resonance imaging (MRI) (3 T
Allegra MRI scanner; Siemens, Erlangen, Germany) before surgery, after which the images were used to locate the lunate sulcus. Electrodes were kept near the position where spike activities were first encountered in a recording track. Characteristics such as high spontaneous activity and brisk on-off responses were taken to indicate that the electrode had entered layer 4 (von der Heydt and Peterhans, 1989; Snodderly and Gur, 1995). Using this strategy, we restricted recordings to the superficial cortical layers.

\section{Results}

The results described herein were on the basis of 114 single-unit records from V2 neurons, 64 from monkey CI and 50 from monkey $\mathrm{CM}$, that responded to some of the 66 angle stimuli in the stimulus set. The significance of the responses was evaluated by comparing the response to each stimulus with the background discharge (Kolmogorov-Smirnov test; $p<0.05$ ). In 91 neurons, there was significant variation among the responses to the various stimuli in the stimulus set (ANOVA; $p<0.05$ ).

\section{Response selectivity to angle stimuli in area $\mathrm{V} 2$}

When we compared the response selectivity of V2 neurons in the angle space, we found that some were indeed selective for certain angle stimuli, as exemplified in Figure 2. Figures 2, $A$ and $B$, shows the 66 angle stimuli in the stimulus set and the response of a V2 neuron (cell 1) to each stimulus in the format of a $12 \times 12$ matrix. This neuron showed statistically significant responses to several stimuli that are indicated by frames around the peristimulus histograms (Fig. $2 B$ ). Strong responses were elicited by an angle stimulus composed of $180^{\circ}$ and $300^{\circ}$ half-lines. In the response profile (Fig. $2 C$ ), the diameters of the disks indicate the magnitudes of the responses to the corresponding stimuli. To determine the optimal angles, we smoothed the profile to avoid picking up local maximums (Fig. $2 D$ ). For cell 1, two neighboring angle stimuli met the criteria of $80 \%$ of the maximal response, which we then used to determine the preferred angles of the neuron in the smoothed profile (see Materials and Methods). We thus judged that cell 1 had one optimal angle (Fig. $2 D$, open circles) formed by $180^{\circ}$ and $300^{\circ}$ half-lines.

To evaluate the specificity of the response selectivity to angle stimuli, we defined the peak response area (Fig. $2 E$, gray regions) (see Materials and Methods). The peak response area of cell 1 extended across two stimuli (4.5\% of the stimuli in the set) around the response to the optimal angle (Fig. 2 E, open circles). Figure $2 F$ shows the actual angle stimuli that elicited responses equal to or larger than half the maximal response; the optimal angle is indicated by a circle. Apparently, cell 1 was sharply tuned to a particular combination of two line components.

Figure $2 G$ shows that, in addition to angle stimuli, cell 1 had selectivity for the direction of the half-lines. This direction selectivity was broad for short half-lines $\left(0.3^{\circ}\right.$ in length), whereas only $180^{\circ}$ produced a strong response for long half-lines $\left(4.0^{\circ}\right.$ in length). Interestingly, there was no response to a half-line of $300^{\circ}$, although it was a component of the optimal angle. This suggests that selectivity for angle stimuli cannot be explained simply by orientation selectivity for the half-lines comprising the angle stimuli. Cell 1 showed weak responses to both short $\left(0.6^{\circ}\right.$ in length) and long ( $8.0^{\circ}$ in length) line segments presented at the center of the CRF (Fig. $2 H$ ).

Figure 3 shows another example, which had an optimal angle stimulus composed of $120^{\circ}$ and $210^{\circ}$ half-lines (cell 2). The peak response area of cell 2 was a single continuous area, extending across five stimuli (Fig. $3 E$ ) $(7.6 \%$ of the stimuli in the set). In contrast to cell 1 , moderate responses were observed to angles 
A

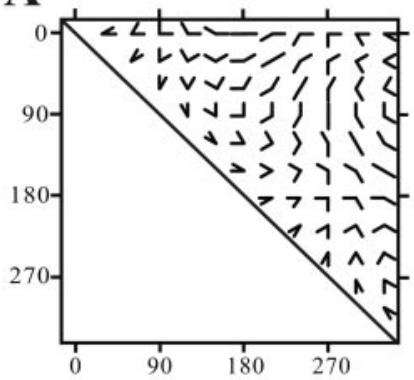

C Response Profile

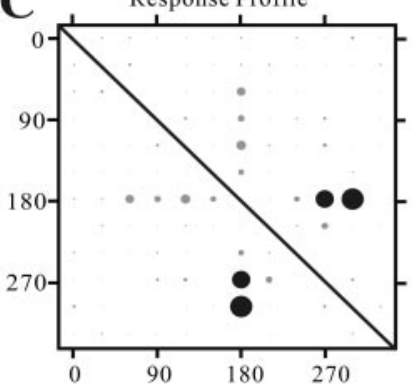

E Response Profile

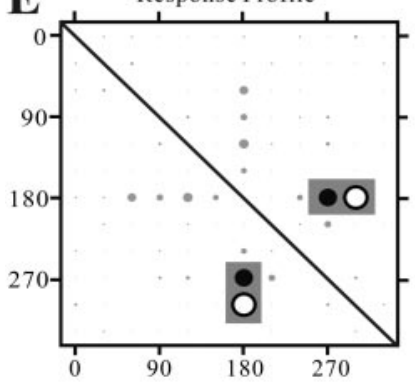

B

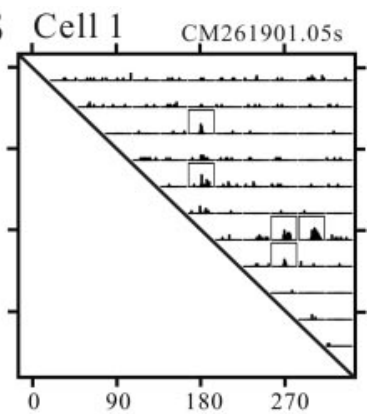

D

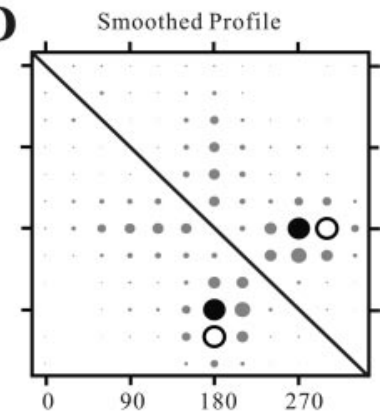

$\mathbf{F}$

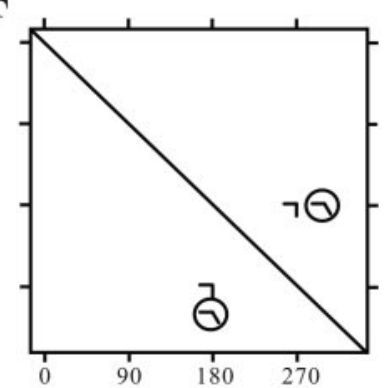

G

$\mathbf{H}$

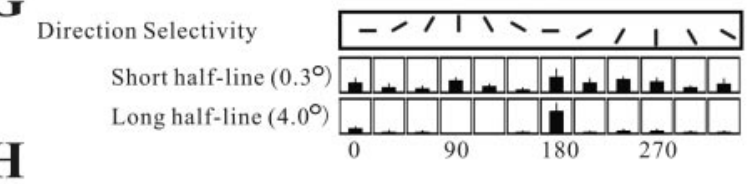

Orientation Selectivity

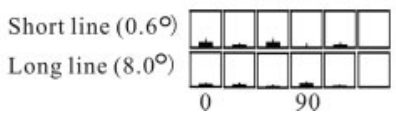

Figure 2. Angle selectivity in a V2 neuron (cell 1). A, Matrix $(12 \times 12)$ containing the 66 angle stimuli. $B$, Responses by cell 1 to each of the angle stimuli shown as peristimulus time histograms (PSTHs) plotted at the position corresponding to the angle stimuli in the stimulus matrix. During a $600 \mathrm{msec}$ period, angle stimuli were presented for $200-400 \mathrm{msec}$. Frames around PSTHs indicate that responses were significantly greater than background responses (Kolmogorov-Smirnov test; $p<0.05$ ). The frame height corresponds to 100 spikes per second; frame width corresponds to $600 \mathrm{msec}$. C, Response profile of the same data in $B$. The magnitude of the response to each angle stimulus is represented as the diameter of disks in the $12 \times 12$ matrix. Black disks indicate responses $\geq 50 \%$ of the maximum. The maximal response was 23.0 spikes per second. Note that the bottom left part is a mirror image of the top right part and that this neuron had only one preferred angle. D, Smoothed response profile of the same data. Open circles indicate responses to the preferred angle. Black disks indicate responses $>80 \%$ of the maximum. The maximal response was 9.2 spikes per second. E, Response profile similar to C in which the open circle indicates the response to the preferred angle. The gray area indicates the peak response area. $F$, Angle stimuli that generated responses $\geq 50 \%$ of maximum are shown in the $12 \times 12$ matrix; the circle indicates the optimal angle stimulus. G, Direction profile measured with short $\left(0.3^{\circ}\right)$ and long $\left(4.0^{\circ}\right)$ half-lines. $H$, Orientation profile measured using short $\left(0.6^{\circ}\right)$ and long $\left(8.0^{\circ}\right)$ lines. Frame height in $G$ and $H$ corresponds to 30 spikes per second.

A

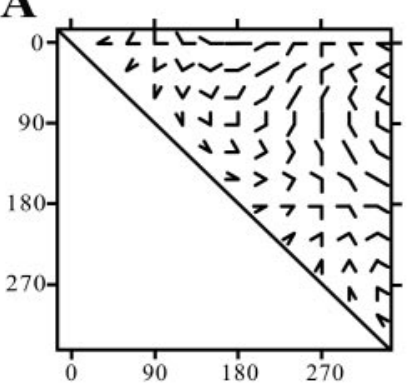

C Response Profile

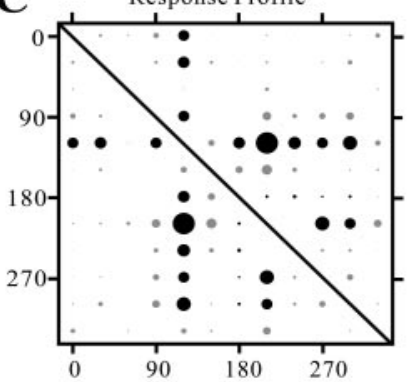

E

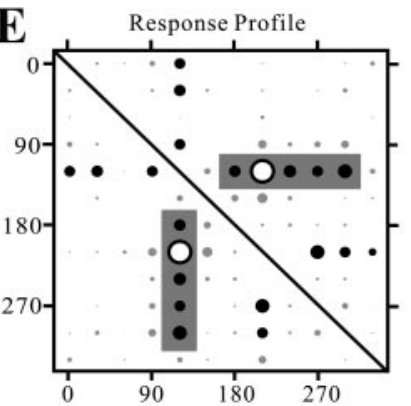

B Cell 2 CM282901.09s

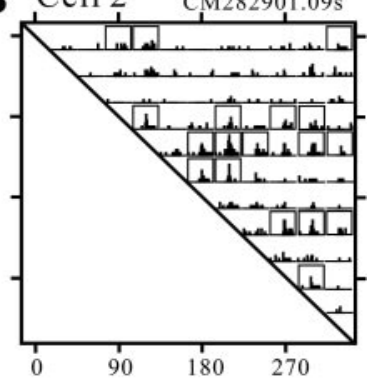

D Smoothed Profile

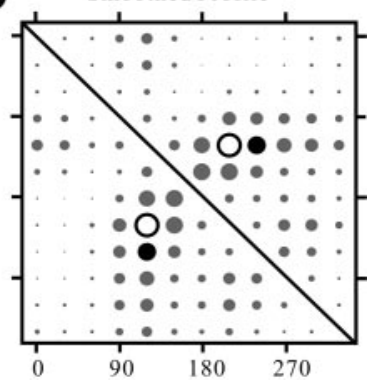

$\mathbf{F}$

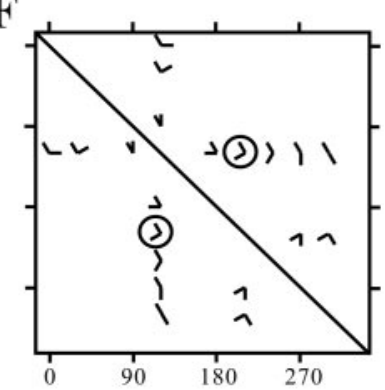

G

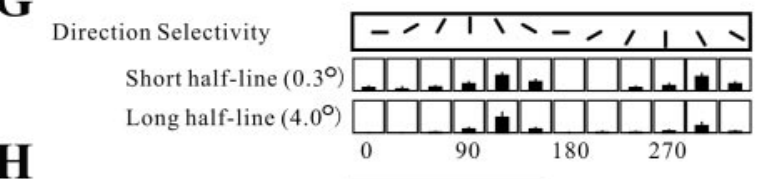

Orientation Selectivity

Short line $\left(0.6^{\circ}\right)$

Long line $\left(8.0^{\circ}\right)$

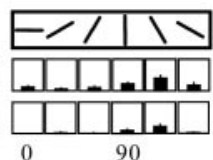

90

Figure 3. Angle selectivity in another V2 neuron (cell 2). The format is the same as in Figure 2. The frame height corresponds to 50 spikes per second $(B, G, H)$. The maximal responses were 17.0 spikes per second $(C, E)$ and 9.5 spikes per second $(D)$.

made up of a combination of the $120^{\circ}$ half-line and several other half-lines, and the peak response area was elongated along the horizontal-vertical axes representing the $120^{\circ}$ half-line component (Fig. $3 E$ ). On the other hand, no elongation of the peak response area was observed along the horizontal-vertical axis representing the $210^{\circ}$ half-line component. Apparently, cell 2 responded to the $120^{\circ}$ line component when it was combined with downward line components $\left(180-300^{\circ}\right)$. The combination with half-line components oriented in other directions not only produced weak responses, but also reduced responses to the preferred line component. The response selectivity of this neuron may therefore be related to the responses to the individual line components, although simple linear summation of those responses could not explain the response selectivity of the neuron. 

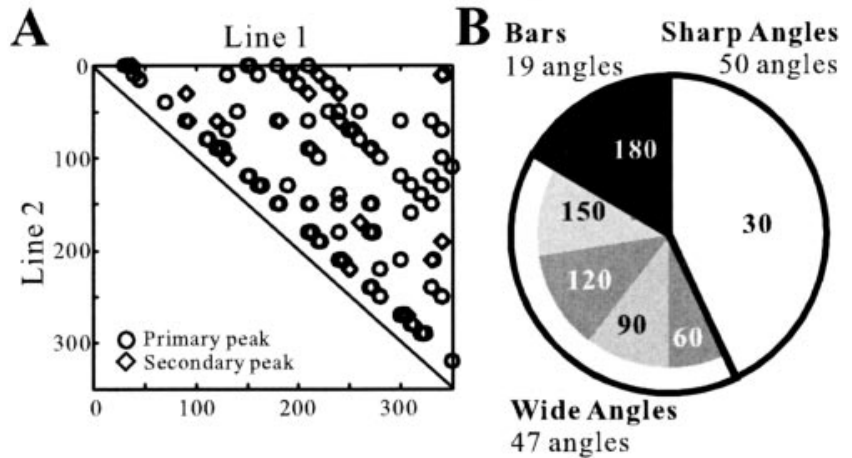

C

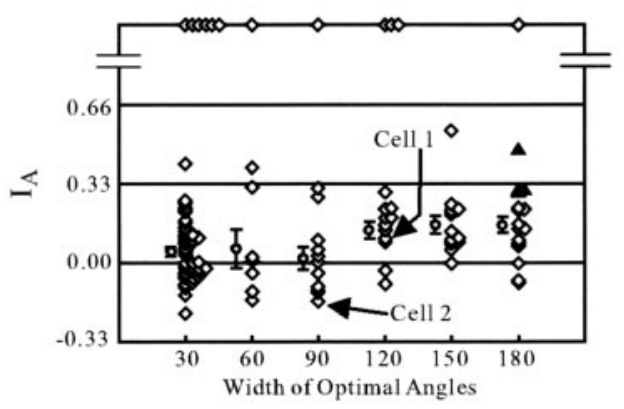

Figure 4. A, Distribution of the preferred angles in the angle space. Horizontal and vertical axes indicate the exact directions of the half-line components of the preferred angle in degrees. Circles denote the primary peaks; diamonds denote the secondary peaks. $B$, Distribution of widths of the preferred angle stimuli. Each number within the circle indicates the width of the preferred angle in degrees. Three classes were distinguished: (1) wide angles, ranging between $60^{\circ}$ and $150^{\circ}(n=47) ;(2)$ sharp angles of $30^{\circ}(n=50)$; and (3) bars corresponding to straight $180^{\circ}$ angles $(n=19)$. C, Angle response index $\left(I_{A}\right)$ of each neuron sorted according to the width of the optimal angles. Filled triangles represent neurons in which the maximal response to the angle stimulus was significantly different from that to the half-lines. Data points at the top of the graph indicate neurons in which a half-line was not sufficient to induce a significant response. Circles and error bars at the left of the data points indicate the average and the SEM.

More likely, this neuron has selectivity to a particular combination of two line components.

Figure $3 G$ shows that cell 2 was also selective for particular half-lines. For short half-lines $\left(0.3^{\circ}\right.$ in length $)$, both $120^{\circ}$ and $300^{\circ}$ induced strong responses, whereas only $120^{\circ}$ produced a strong response for long half-lines $\left(4.0^{\circ}\right.$ in length). That the selectivity tended to be sharper for long half-lines probably indicates that they activated inhibitory mechanisms. Interestingly, there was no response to the $210^{\circ}$ half-line, although it was a component of the optimal angle. Again, this suggests that selectivity to angle stimuli cannot be explained simply by the orientation selectivity for the half-lines comprising the angle stimuli. Cell 2 also responded to line segments presented at the center of the CRF, although the response amplitudes were smaller than the maximal response (Fig. $3 H$ ). Cell 2 showed significant orientation selectivity to both short ( $0.6^{\circ}$ in length) and long ( $8.0^{\circ}$ in length) line segments.

In total, we obtained 91 primary peaks and 25 secondary peaks (see Materials and Methods) from 91 angle-selective V2 neurons. We next investigated the properties of these neurons in more detail with the aim of obtaining clues about the neuronal mechanisms by which angle features are extracted.

\section{Distribution of optimal angle stimuli in area V2}

We observed diverse angle selectivity in area V2. Figure $4 A$ shows the distribution of the optimal angles within the angle space, with the exact direction of each half-line given on the axes. We found no bias in the distribution of the optimal angles, and the orienta- tion of the angle components for both the primary (circle) and secondary (diamond) peaks was distributed over a wide range. Figure $4 B$ shows the distribution of widths of the optimal angles in the primary and secondary peaks. Of 116 optimal angles, 47 $(40.5 \%)$ were "wide" angles, ranging between $60^{\circ}$ and $150^{\circ}$. The broad distribution of optimal widths in this class indicates that V2 neurons represent a variety of angular components within contour stimuli. Fifty (43.1\%) were "sharp" $\left(30^{\circ}\right)$ angles. Because the total length and brightness of the angle stimuli were different from those of the individual half-lines, responses elicited by each were not directly comparable. Consequently, we were unable to quantitatively determine whether the selectivity of these neurons for $30^{\circ}$ angles reflected specific tuning to very sharp angles or broad tuning to single line-ends. The remaining 19 angles $(16.3 \%)$ were long "bars" $\left(180^{\circ}\right)$, i.e., straight lines $6.0^{\circ}$ or $8.0^{\circ}$ in length that passed through the CRFs. Although these neurons were appropriate for conveying information about the orientation of straight contours passing through the CRF, they accounted for a surprisingly small fraction of V2 neurons. In 14 neurons, the primary and secondary peaks belonged to different classes of angles. Considering this, 41 (36.0\%) neurons were selective to wide angles $\left(60-150^{\circ}\right), 45(39.5 \%)$ to sharp angles $\left(30^{\circ}\right)$, and $19(16.7 \%)$ to long bars. Notably, neurons selective for angle stimuli also responded to straight contours with submaximal responses, and 47 neurons (51.6\%) showed significant orientation selectivity for the straight contours. This means that the responses to line segments might be overestimated if the response selectivity was studied with only straight lines.

As observed with cells 1 and 2, many V2 neurons showed clear responses to the half-lines that we used to form angle stimuli. We therefore compared the maximal responses to angle stimuli with those to half-lines (Fig. $4 C$ ). The angle response index $\left(I_{\mathrm{A}}\right)$ fell between 0.33 and -0.33 in most cases, indicating that the maximal responses to angle stimuli and half-lines were generally comparable. Comparison of firing rates of the maximal responses to angle stimuli and those to half-lines showed that only four neurons exhibited significant differences (Kolmogorov-Smirnov test; $p<0.05$ ), all of which preferred bars (Fig. $4 C$, filled triangles). Mean values of indices showed a weak tendency for the angle response index to be nearly zero for angles $<120^{\circ}$ in width and to be larger than zero for other angles and bars. For wide angles $120^{\circ}$ and $150^{\circ}$ in width and bars, the indices were significantly larger than that for sharp angles $(t$ test; $p<0.05)$. To summarize, V2 neurons are sensitive not only to angle stimuli but also to half-line stimuli. Nevertheless, when contour stimuli pass through the receptive fields of neurons, these V2 neurons can differentiate the width and orientation of the angles. We conclude that a fairly large number of area V2 neurons are angle selective and involved in the representation of various angles in addition to straight line segments.

\section{Peak response area in response profiles}

We next examined the tuning width of the angle selectivity using the size of the peak response area as an index. The distribution of the size among 116 optimal angles ranged from 1 to 56 (Fig. 5). On average, the size was 8 of 66 stimuli ( $12.1 \%$; median; $n=116)$ (Fig. 5A, downward arrow), indicating moderate tuning of the angle selectivity, although the size of the peak response area was $<4$ for 30 optimal angles (25.9\%). Thus, a considerable number of neurons showed highly selective responses to a particular angle for both primary and secondary peaks (Fig. 5A). Among the three classes of optimal angle, broad tuning of the angle selectivity was mainly observed in the sharp and wide classes, whereas the bar 


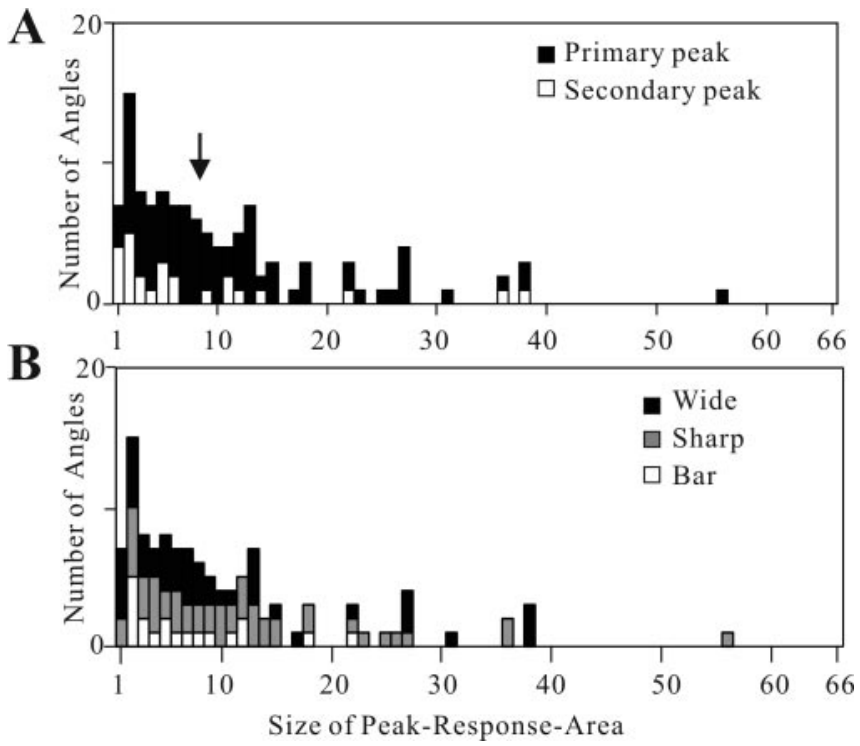

Figure 5. Distribution of the sizes of peak response areas. The downward arrow indicates the median $(n=116)$. The distribution was divided with respect to two sets of properties: primary or secondary peaks $(A)$, and wide angles, sharp angles, or bars $(B)$.

class had relatively small peak response areas (Fig. 5B), which suggests that V2 neurons selective for a long straight bar are sensitive to orientation and small distortions in contour lines.

We also analyzed the spatial pattern of the distribution of the responses in the response profile. As shown by the responses of cell 3 (Fig. 6A), large peak response areas tended to have particular axes of elongation that provide information about angle features important for inducing selective responses. We assumed four situations. (1) If a neuron responded to each half-line of the optimal angle rather than to a particular combination of two components, it should respond to any stimulus containing either half-line having the optimal orientation, and the distribution of the responses would elongate along the horizontal-vertical axes representing the line components (Fig. 6C). (2) If a neuron was selective for angle width, regardless of the direction of the individual components, the response distribution would elongate along a diagonal axis from left top to right bottom (Fig. 6D). (3) If a neuron was selective for angle orientation, regardless of the direction of the individual line components, the response distribution would elongate along a diagonal axis from right top to left bottom (Fig. 6E). (4) Otherwise, the responses distribution would be evenly spread around the optimal angles, indicating that the selectivity was dependent mainly on the combination of the two line components. Here, we examined which of these patterns best reflected the selectivity of each neuron.

We first evaluated the extent of the elongation, i.e., the number of stimuli inducing responses equal to or larger than half the maximal response, along the four axes centered at the optimal angle, as shown in Figure $6 C-E$ (gray regions). We then compared these numbers to characterize the distribution of the responses (Fig. 7). Because the response matrix was discontinuous at the diagonal line, which corresponds to angle stimuli $0^{\circ}$ in width, we used an oblique version of the response profile (Fig. $6 B-E$, dotted rectangular area) consisting of the same set of angle stimuli. The maximum scale along the axis for the line components and the angle orientation was 11 and that for the angle width was 12 . We will refer to the larger of the horizontal-vertical axes as the "primary axis" and to the smaller as the "secondary axis." In the case of cell 3 , the extent of the elongation was 8 along the horizontal axis representing the $120^{\circ}$ half-line component, 11 along the vertical axis representing the $240^{\circ}$ half-line component, 5 along the diagonal axis representing the angle width $\left(120^{\circ}\right)$, and 5 along the diagonal axis representing the angle orientation $\left(0^{\circ}\right)$. Figure $6 \mathrm{~F}$ shows the distribution of the extent of the elongation along the four axes for the 116 optimal angles. The greatest elongation was observed mainly along the primary horizontal-vertical axes representing one line component of the optimal angles.

We next compared the extent of elongation along three axes: the axis for the line component (either primary or secondary axis), the axis for angle width, and the axis for angle orientation. In 29 of 116 angles $(25.0 \%)$, the extent of elongation within the peak response area was less than three along all four axes, and their responses were too specific to evaluate elongation (e.g., cell 1) (Fig. 6F, gray column). These responses appear highly dependent on the combination of the two half-line components. For the remaining 87 angles, the relative extent of elongation along the three axes was characterized by computing the barycenter of the three values (Fig. 7) (see Materials and Methods). There was a clear tendency for the distribution of the responses to elongate along the primary axis for the line component.

When the extent of elongation along one axis was equal to or greater than twice those along the other two axes, we deemed there to be anisotropy and refer to this axis as the "axis of elongation." In the case of cell 3, the vertical-horizontal axis representing the $240^{\circ}$ half-line was the axis of elongation. Twentyseven angles $(23.3 \%)$ had an axis of elongation, all of which represented one component of the optimal angles (Figs. $6 \mathrm{~F}$, first row, black column, $7 A$, open symbols). For four of these angles, all of which were bars, the secondary horizontal-vertical axis also met the criteria for an axis of elongation (Figs. $6 F$, second row, black column, $7 B$, open symbols). We observed no neuron that had a diagonal axis of elongation, suggesting that area V2 does not represent the width or orientation of an angle, per se. This means that the responses to angle stimuli were dependent to a large extent on the responses to one optimal half-line; however, the specific combination of two line components was also necessary to maximally activate these neurons. The remaining $60 \mathrm{op}-$ timal angles $(51.7 \%)$ had no axis of elongation, although they had relatively large peak response areas, indicating that they are sensitive to a specific combination of two half-line components, but with relatively broad angle tuning.

Collectively, the results summarized above suggest that specific combinations of two half-line components are required to elicit optimal responses in most V2 neurons, regardless of tuning width (Fig. 7C). Most of the sharp (33 of 50; 66.0\%) and wide (24 of $47 ; 51.1 \%$ ) classes had no axis of elongation, although many had large peak response areas. One-fourth of the wide and sharp classes had an axis of elongation only along the primary horizontal-vertical axes. The bar class was split into two types: one sensitive to a long straight line, i.e., an angle $180^{\circ}$ in width, that was assigned to either the "specific responses" or "no axis" class depending on the size of peak response area (11 of $19 ; 57.9 \%)$, and another sensitive to one or both of the half-line components ( 8 of $19 ; 42.1 \%)$.

\section{Consistency between responses to each line component and optimal angles}

To determine the contributions made by the individual line components, we examined the relationship between angle selectivity and the responses to the individual half-line components of the angle stimuli ("direction profiles"). Figure $8 \mathrm{~A}$ shows the direc- 
A

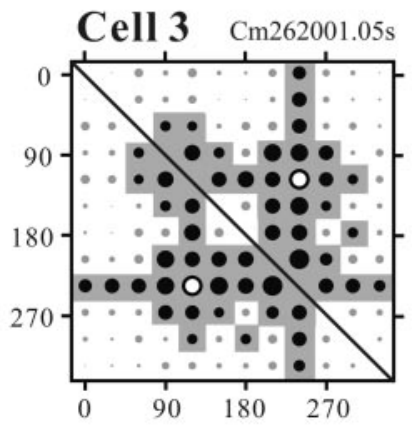

B

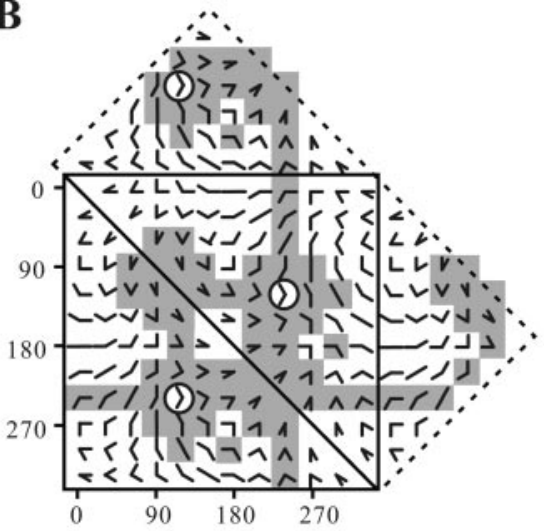
30

Axis for Line Component (Primary)

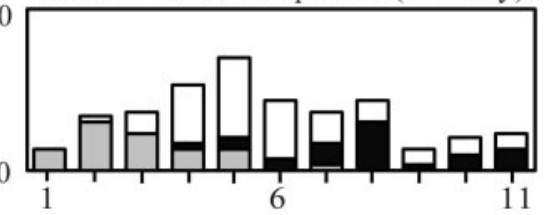

Axis for Line Component (Secondary)

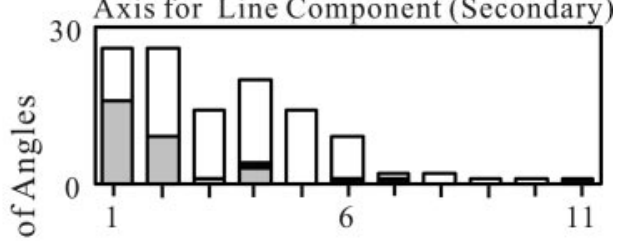

Axis for Angle Width

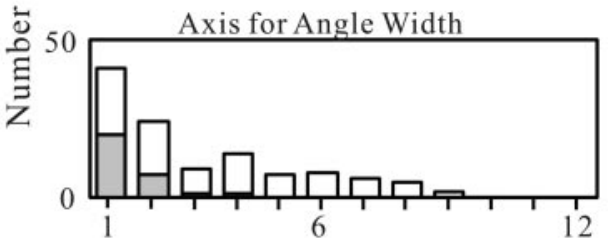

Axis for Angle Orientation

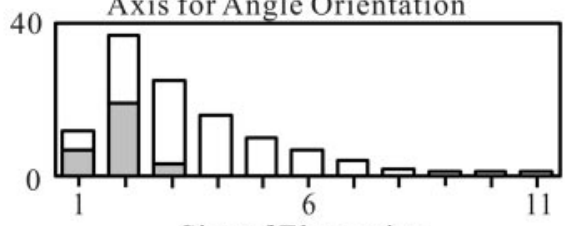

Size of Elongation

Figure 6. Elongation of the distribution of responses in the angle space was given by the number of stimuli along the four axes centered at the optimal angle. $A$, Distribution of responses equal to or larger than half the maximal response (gray region) in a V2 neuron (cell 3). The format is the same as in Figure 2E. B, Extended version of the matrix of the angle space. Note that the oblique matrix shown by the broken line contains the same angle stimuli as the original matrix. The gray region contains angle stimuli inducing responses equal to or larger than half the maximum; open circles indicate the optimal angle. $(-E$, The four axes centered at the optimal angle in the oblique matrix (gray region). Only stimuli inducing the responses equal to or larger than half the maximum are indicated. The extent of the elongation is given by the number of these stimuli in the gray regions. C, Axes for the line components of the optimal angle. D, Axis for the angle width of the optimal angle. $E$, Axis for the angle orientation of the optimal angle. $F$, Distribution of the extents of elongation along the four axes for 116 optimal angles. For the horizontal-vertical axes, the larger axis is referred to as the primary axis, and the smaller is referred to as the secondary axis. The black column represents the optimal angles in which the extent of elongation met the criteria for axis of elongation (see Results). The gray column represents those in which the extent of elongation within the peak response area was less than three along all four axes.

tion profiles of four neurons that had from one to three peaks for preferred directions. Figure $8 B$ shows the relationships between the response amplitudes of the primary peaks and that of the secondary peaks. A secondary peak was assigned for further analysis if the amplitude was equal to or larger than half the maximum and was separated from the primary peak by responses smaller than half the maximum (Fig. $8 B$, filled symbols), and a third peak could be assigned in the same way. For nine neurons, response amplitudes next to the primary or secondary peaks were $>95 \%$ of the peak response and regarded as parts of a broad preferred direction (e.g., secondary peak of cell 3). Peaks are indicated by black dots in Figure 8 A. Of 91 neurons, 33 (36.3\%) had a single peak, $33(36.3 \%)$ had two peaks, and $15(16.5 \%)$ had three peaks; half-lines did not induce significant responses in the remaining 10 neurons $(8.9 \%)$ ). Direction profiles were then classified into four groups: (1) "bimodal/trimodal $\left(180^{\circ}\right)$ " selectivity with two or three peaks, including a pair $180^{\circ}$ apart, which might be appropriate for representing straight contour lines; (2) bimodal/trimodal selectivity with two or three peaks without a pair $180^{\circ}$ apart, which might be appropriate for representing angles; (3) "unimodal" selectivity with only one peak, which might be appropriate for representing line-ends; and (4) "no (significant) response" to half-lines. Interestingly, when neurons were classified according to their optimal angles, all four types of direction profile were observed in all three of the resultant groups (Fig. $8 \mathrm{C}$ ). For example, some neurons had bimodal $\left(180^{\circ}\right)$ selectivity to individual line components and preferred a wide angle rather than straight lines. Consequently, the class of the direction profile was not sufficient to explain the angle selectivity.

In Figure $8 \mathrm{~A}$, arrows indicate the directions of two components of the optimal angle stimulus. Cells 3 and 6 had multiple peaks, which were consistent with the two components of the optimal angle. Because the difference between the two preferred directions was not $180^{\circ}$ for each neuron, these preferred directions did not represent the orientation preference of the neurons but likely the two half-line components of the optimal angle. In contrast, cell 1 had only one preferred direction, which was consistent with one of the components of the optimal angle (Fig. $8 \mathrm{~A}$, black arrow); no response was observed for the other line component (white arrow). Cell 2 had two preferred directions: one consistent with a component of the optimal angle (black arrow) and the other not (white arrow). Figure $9 A$ shows a chart of the consistency between the direction profile and the optimal angles for all neurons that preferred wide angles. In this chart, we compared the direction of each component of the optimal angle (downward arrowheads) and the peak (black thick line) or the peak regions (gray thick line) in which the responses were $\geq 50 \%$ of the maximum in the direction profile. We commonly observed that the direction of the line components of the optimal angle was consistent with the peaks (black arrowheads) or the peak regions (gray arrowheads), as indicated on each line. For the majority of those matching the peak region, the direction of the line compo- 
A

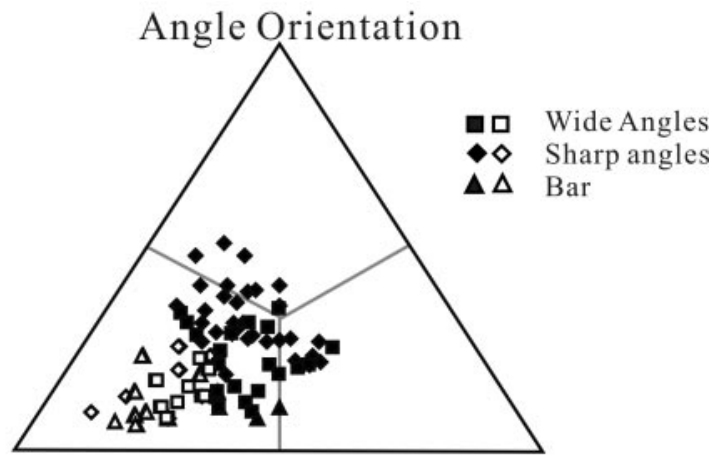

\section{Line Component (Primary) \\ Angle Width}

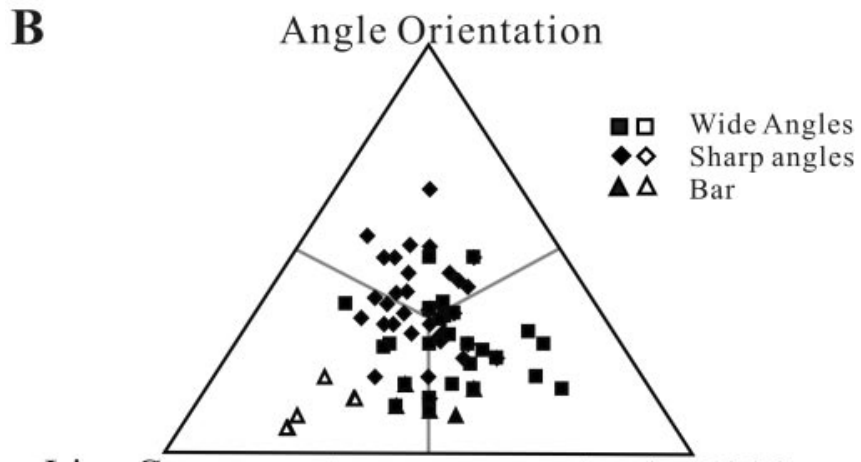

Line Component (Secondary)

C (\%)

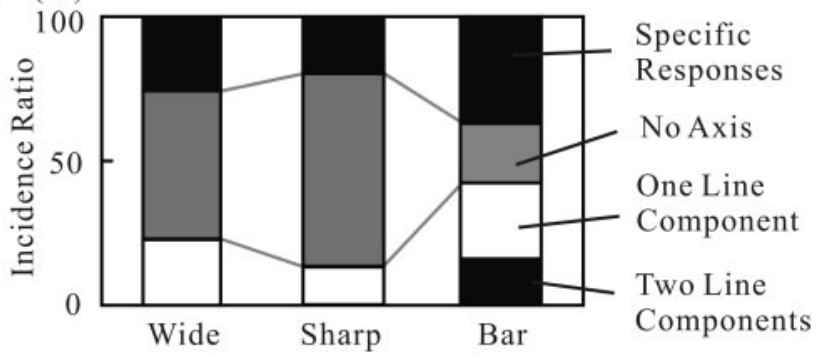

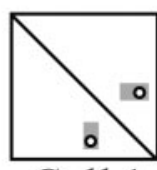

Cell 1

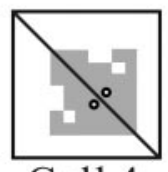

Cell 4

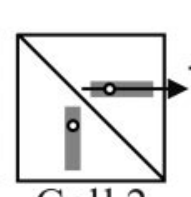

Cell 2
Angle Width

Figure 7. A, Comparison of the extent of elongation along three axes representing the primary line components, angle width, and angle orientation, respectively. Individual points show the barycenter of the three values for wide angles (squares), sharp angles (diamonds), and bars (triangles). Open symbols meet the criteria of the axis of elongation (see Results). B, Comparison of the extent of elongation along the three axes representing the secondary line components, angle width, and angle orientation, respectively. The format is the same as in $A$. C, Classification of the optimal angles with respect to the presence or absence of an axis of elongation. In the "Two Line Components" class, the distribution of responses had two axes of elongation representing the two line components of the optimal angles. In the "One Line Component" class, the distribution of responses had one axis of elongation representing one line component of the optimal angles. There was no elongation in the "No Axis" class and a small peak response area in the "Specific Responses" class. The incidence ratio is shown for each category of optimal angle: wide, sharp, and bar. The insets show examples of the response profiles for each of the above four classes: the gray areas indicate the peak response area, open circles indicate the optimal angle, and the arrow indicates the axis of elongation.
A
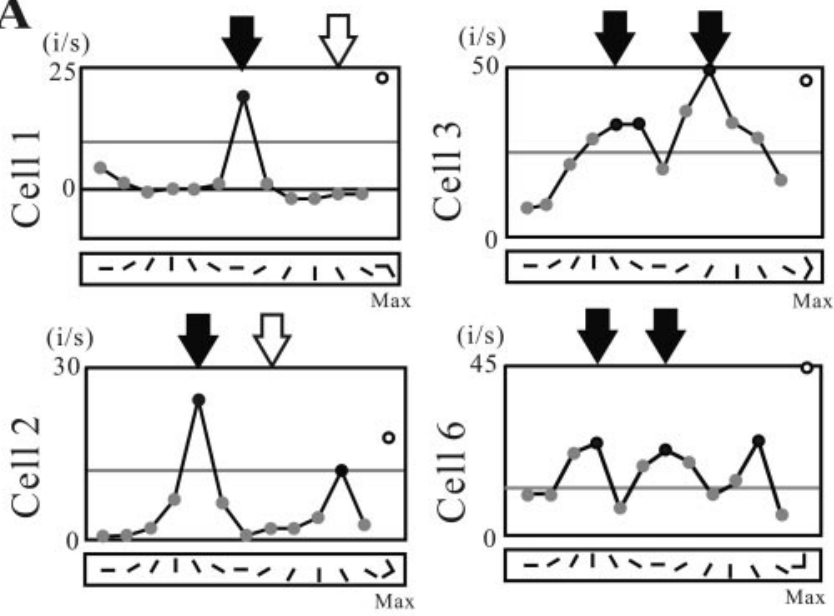

B

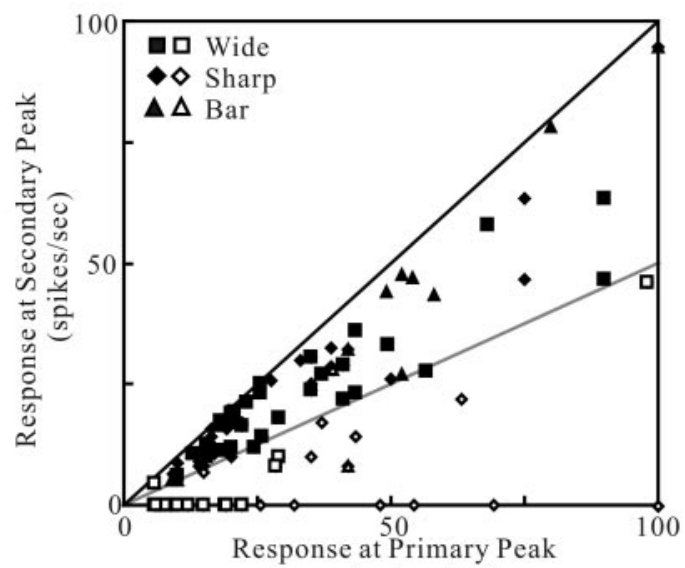

(spikes/sec)

C

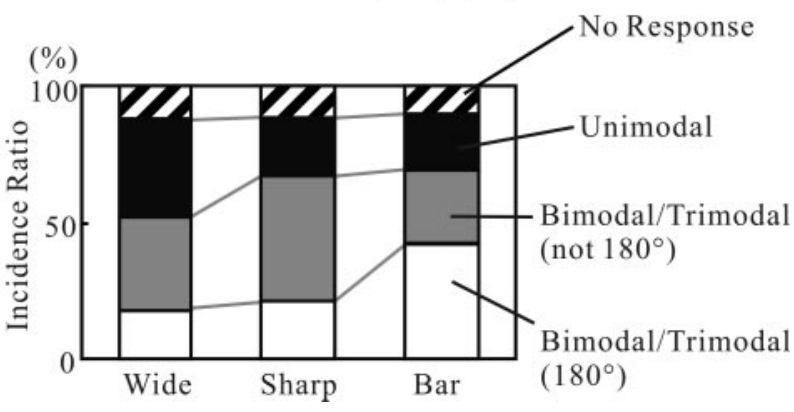

Figure 8. Direction selectivity for half-lines. $A$, Direction profiles of four neurons. Line charts show the responses to individual half-lines oriented in 12 directions separated by $30^{\circ}$. Black dots indicate the peak responses, gray lines indicate the $50 \%$ of the maximum point, and the open circles at the right of the direction profile indicate the maximal responses to the angle stimuli. When response amplitudes next to the primary or secondary peaks were $>95 \%$ of the peak response, they were regarded as parts of a broad preferred direction (e.g., secondary peak of cell 3). Arrows indicate the directions of the line components of the optimal angle, which may be consistent (black arrows) or inconsistent (white arrows) with the peak responses to half-lines. $B$, Relationships between response amplitudes of the primary and secondary peaks in three categories of optimal angle: wide (squares), sharp (diamonds), and bar (triangles). When only single peaks were observed, data were plotted on the horizontal axis. When no significant responses were induced by any half-lines, the data were omitted from the figure. The gray line indicates the point at which the height of the secondary peak is half that of the primary peak. Secondary peaks $<50 \%$ of the maximum (open symbols) were discarded from further analysis. C, Classification of 116 optimal angles with respect to the direction selectivity to half-lines. Each

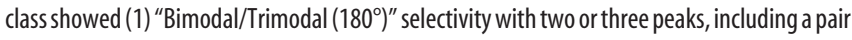
$180^{\circ}$ apart; (2) "Bimodal/Trimodal" selectivity with two or three peaks, without a pair $180^{\circ}$ apart; (3) "Unimodal" selectivity with only one peak; and (4) "No (significant) Response" to half-lines. Shown are the incidence ratios for each of the three categories of optimal angles: wide, sharp, and bar. 
A

\section{Bimodal/Trimodal $\left(180^{\circ}\right)(\mathrm{n}=8)$}

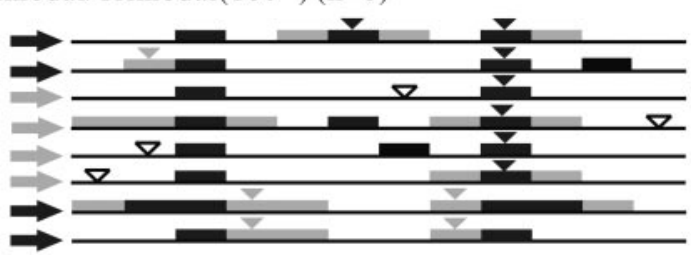

Bimodal/Trimodal (not $\left.180^{\circ}\right)(\mathrm{n}=16)$

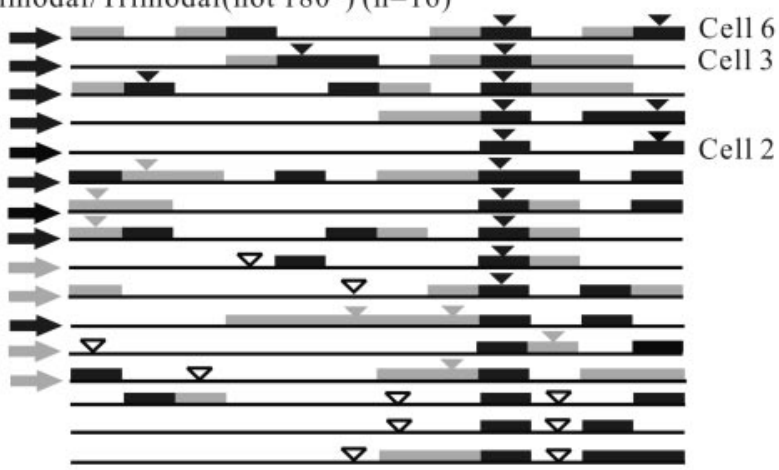

Unimodal $(\mathrm{n}=18)$

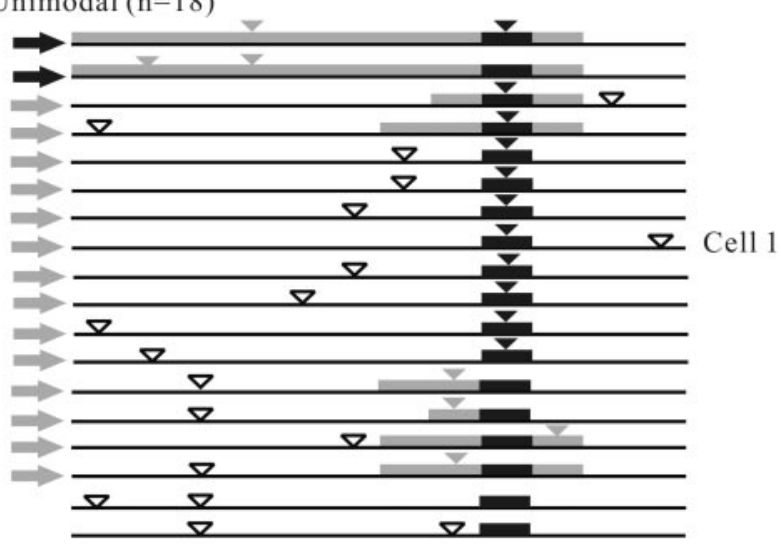

No Response $(\mathrm{n}=5)$

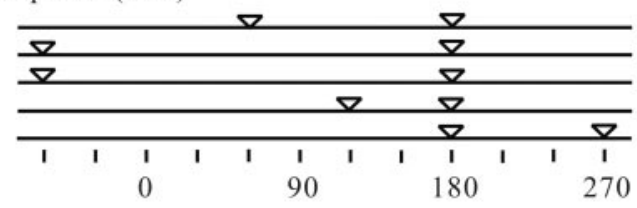

Direction of Half Line (degree)

B

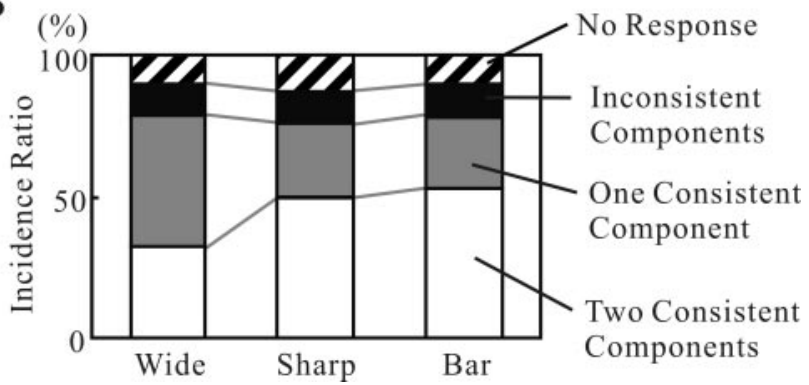

Figure 9. Consistency between the direction selectivity for half-lines and components of optimal angles. $A$, Chart of the consistency among all neurons that preferred wide angles. Cells were divided into four groups with respect to the bimodality of the direction profile to half-lines. Each horizontal line corresponds to one optimal angle. Thick black lines represent the peak in the direction profile. Thick gray lines represent the peak regions in which response amplitudes are $\geq 50 \%$ of the maximum. Direction was aligned with the direction corresponding to one peak and assigned a value of $180^{\circ}$. Downward arrowheads indicate the directions of the line nent was next to the peak ( 15 of $19 ; 78.9 \%$ ). We observed that in 15 cases $(31.9 \%)$ both of the line components of the optimal angle were consistent with the peaks or the peak regions (black rightward arrows at the left), especially among the bimodal/trimodal classes; in 22 cases $(46.8 \%)$, one line component of the optimal angle was consistent (gray rightward arrows at the left), mainly in the unimodal class.

The above results suggest that for most angle-selective V2 neurons, the response to the optimal angle reflects the specific combination of two line components. In many of these neurons, the response to the optimal angles also reflected the signal representing one half-line component, with the other acting as a modulator. This tendency was particularly clear in the wide class (Fig. $9 B$ ). As shown in Figures $4 C$ and $8 A$, the amplitudes of the maximal responses to angles and half-lines were comparable with one another. The result also indicates that modulatory inputs are involved in the formation of angle selectivity and that simple summation of responses to each half-line component was not enough to explain the response selectivity of a neuron.

Stimulus sites and eye positions during stimulus presentation It was essential in this study that stimuli form angles at the center of the CRFs. If angles were presented near the border of a CRF, a combination of the orientation selectivity and end-stop inhibition might have produced pseudo-response selectivity to angle stimuli. Indeed, Heider et al. (2000) reported that half of the end-stop neurons in area V2 were single end-stopped and that several of these responded to one right corner but not to its opposite. To avoid such pseudo-angle selectivity, we took several precautions when determining the centers of CRFs.

We first determined the borders of the CRFs using the minimum response method (Barlow et al. 1967) with an optimal short bar. Figure $10 \mathrm{~A}$ shows the CRF of cell 2, the responses of which to angle stimuli are shown in Figure 3. A dot indicates the center of the CRF, where the angles were formed. We next confirmed the centers of the CRFs quantitatively by presenting a short line segment $\left(0.5^{\circ}\right.$ in length $)$ at the optimal orientation $\left(0^{\circ}\right.$ in exact orientation for cell 2) in a $5 \times 5$ matrix of $0.5^{\circ}$ intervals around the center of the CRF (Fig. $10 \mathrm{~B}$ ). Note that the center of the response matrix corresponded to the location of angle stimuli and should induce maximal responses. If the maximal response was not observed at the center of the response matrix, the CRF was determined again. We then studied the directional selectivity of halflines short enough to be presented within the CRFs $\left(0.3^{\circ}\right.$ in length). If the short half-line of either the optimal or opposite direction $\left(0^{\circ}\right.$ and $180^{\circ}$ for cell 2 ) (Fig. $3 G$ ) induced only a weak response, we assumed that stimuli were presented near the border of the CRF, so that one short line failed to activate the neuron or activated end-stop inhibition of the neuron. In such cases, we determined the CRF again.

The stability of the animals' fixation was another important factor, because the rotation task strongly required accurate fixation throughout. The mean and SD of the eye positions recorded

$\leftarrow$

components of optimal angles; black arrowheads are consistent with the peak of the direction profile, gray arrowheads are consistent with the peak region, and white arrowheads are inconsistent with peaks and peak regions. Rightward arrows at the left indicate the classification with respect to consistency; black arrows indicate that both two-line components were consistent; gray arrows indicate that only one line component was consistent. B, Classification of 116 optimal angles with respect to the consistency: (1) "Two Consistent Peaks"; (2) "One Consistent Peak"; (3) "Inconsistent Peaks"; and (4) "No (significant) Response" to half-lines. Shown are the incidence ratios for the three categories of optimal angles: wide, sharp, and bars. 
A
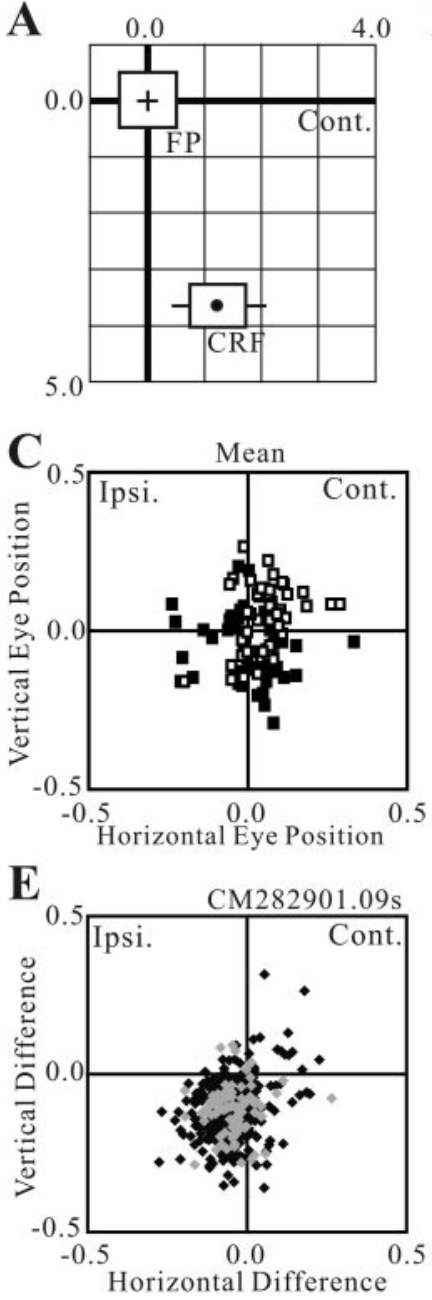

B
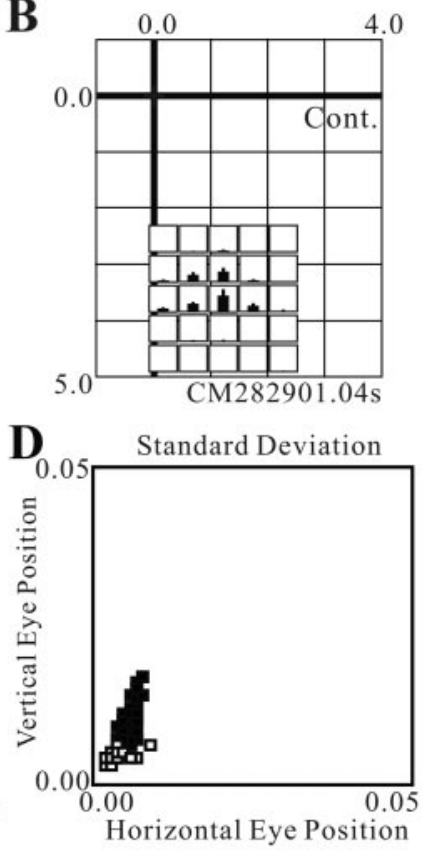

$\mathbf{F}$

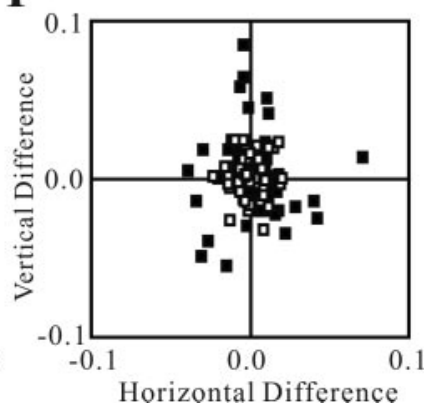

Figure 10. Control of eye position during stimulus presentation. A, CRF of a V2 neuron (cell 2). Optimal orientation was $0^{\circ}$. The dot indicates the center of the CRF, where angles were formed by combining two half-lines. FP indicates the fixation mark and the extent of the $1.0^{\circ} \times$ $1.0^{\circ}$ fixation window. The axes are calibrated in degrees. $B$, Quantitative examination of the CRF. The location of a short $\left(0.5^{\circ}\right)$ line segment was shifted vertically and horizontally in $0.5^{\circ}$ steps, and response magnitudes are shown as the height of the bar in each box of the $5 \times 5$ response matrix. The vertical line on top of each bar indicates the SEM of the response. The response matrix is superimposed on the same coordinates used in $A$, and the position of each box corresponds to where the stimulus was presented. The center of the matrix corresponds to the center of the CRF shown in $A$. Frame height corresponds to 50 spikes per second. C, D, Mean $(C)$ and SD $(D)$ of eye positions during the recordings of each neuron $(n=91)$. $E$, Mean eye positions during stimulus presentation in 528 trials ( 66 angle stimuli $\times 8$ repetitions) in a recording session from cell 2. Gray diamonds represent trials in which the response was $>50 \%$ of maximum, and black diamonds represent those in which the response was $<50 \%$ of maximum. $F$, Differences between the mean eye positions during trials in which responses were greater than half maximum and those in which responses were less than half maximum. Axes are calibrated in degrees for all panels. The different symbols represent the two monkeys in $C, D$, and F. Cont., Contralateral side; Ipsi., ipsilateral side.

during presentation of the angle stimuli for all 114 neurons (Fig. $10 C, D)$ indicated that the animals' fixation was well restricted to within the middle fixation window $\left(1.0^{\circ} \times 1.0^{\circ}\right)$. The eccentricity of the CRFs ranged from $1.6^{\circ}$ to $5.4^{\circ}$, and the square root of their extent was $0.94^{\circ} \pm 0.03^{\circ}$ (mean \pm SEM; $0.43-1.81^{\circ} ; n=114$ ). Considering the accuracy of our CRF mapping and the accuracy of the monkeys' fixation, we are confident that the angle stimuli used in the present study passed through the center of the CRFs, as we intended.

We also considered the fact that response amplitude may be

affected by small variations in eye position during presentation of the angle stimuli. Figure $10 E$ shows the eye positions during each of 528 stimulus presentations ( 66 stimuli $\times 8$ repetitions) in a recording session from cell 2 . When we divided these eye positions into two groups according to the magnitudes of the corresponding responses and then compared their means, we found little difference. When angle stimuli induced responses $>50 \%$ of maximum, mean eye position was $-0.039^{\circ} \pm 0.007^{\circ}$ horizontally $(n=88)$ and $-0.109^{\circ} \pm 0.008^{\circ}$ vertically $(n=88)$; when they induced responses $<50 \%$ of maximum, it was $-0.049^{\circ} \pm 0.003^{\circ}$ horizontally $(n=440)$ and $-0.115^{\circ} \pm 0.004^{\circ}$ vertically $(n=$ 440 ). Figure $10 F$ summarizes this analysis for all 114 neurons. That in most cases the difference was $<0.05^{\circ}$ for both the horizontal and vertical directions provides further evidence that angle selectivity in V2 neurons cannot be explained as pseudo-angle selectivity generated by asymmetric deviation of eye position and stimulus location relative to the CRF.

\section{Discussion}

In the present study, we systematically examined the responses of V2 neurons to a set of 66 angle stimuli. We found that a fairly large number of $\mathrm{V} 2$ neurons selectively responded to angles. Notably, one-fourth of them showed highly selective responses to a particular angle. For most angle-selective V2 neurons, the response to the optimal angle reflected the specific combination of the two line components. The present result is consistent with the idea that signals encoding the orientation and location of individual line components converge on V2 neurons and that extraction of angle features may start in area V2.

\section{Response selectivity to angle stimuli in area $\mathrm{V} 2$}

It has been believed that, like V1 neurons, V2 neurons are selective for the orientation of lines or edges; neurons selective for angle-like stimuli were thought to be rather rare (Kobatake and Tanaka, 1994; Hegde and Van Essen, 2000; Heider et al., 2000). We found, however, that a fairly large number of V2 neurons selectively responded to angles. There are several possible reasons for the discrepancy between the present findings and those of previous studies. First, the present study is the most detailed and systematic assessment of response selectivity to angle stimuli in area V2 reported so far. Previous studies used a smaller number of angle stimuli. Hegde and Van Essen (2000), for example, used 24 angles ( 4 orientation $\times 3$ widths $\times 2$ sizes) in their measurements. Considering the highly selective neurons observed in this study, such a discrete survey could easily miss finely tuned angleselective responses and might therefore underestimate angle selectivity. Second, we used large angle stimuli that passed through the CRFs, whereas previous studies presented angle stimuli as a single object within the CRFs. Because angle selectivity was strongly related to the directional selectivity of the half-lines, which was more evident when using long half-lines, our use of large stimuli favored the revelation of angle selectivity. Third, one-half of our sample showed significant orientation selectivity for long bars, although the responses were submaximal. This means that if we studied only selectivity for straight lines, a large fraction of our sample would be classified as selective for line segment orientation. Our finding is therefore consistent with the previous results indicating that $\mathrm{V} 2$ neurons are orientation selective. Finally, we concentrated on sampling from the superficial layer to increase our chances of discovering the outcome of information processing within this area that in turn would be transmitted to higher cortical areas.

We also found that a small fraction of the V2 neurons studied 
preferred straight lines passing through the CRFs. They were very sensitive to orientation and distortion of line or edges, and their response selectivity was sharper than that of other angle-selective neurons. We suggest that these neurons are suitable for precisely differentiating straight contours from curvatures, angles, and junctions.

Our study shows that the amplitudes of the maximal responses to angles and half-lines are comparable with one another. Therefore, it is unlikely that angle-selective V2 neurons act as specific detectors of angle stimuli. Among the continuous and closed contour lines on the surfaces of objects, however, V2 neurons show a great potential to represent angle width and distinguish angles from straight contour lines.

\section{Responses to angle stimuli in the primary visual cortex}

So far, angle-selective responses have not been reported in area V1 of the monkey. In area 17 of the cat, some neurons selectively respond to curvatures, chevrons, and sharp angles (Heggelund and Hohmann, 1975; Hammond and Andrews, 1978; Dobbins et al., 1987, 1989; Versavel et al., 1990; Shevelev et al., 1998), and some useful mechanisms for extracting angles were evident. For instance, Hubel and Wiesel (1965) suggested that deformation of optimal bar stimuli that avoids activation of end-stop inhibition may result in strong responses to obtuse angles. Sillito et al. (1995) suggested that facilitation caused by recurrent excitation from orthogonal gratings in the surrounding annulus may represent discontinuity of line segments. Das and Gilbert (1999) suggested that inhibitory interaction between nearby neurons having orthogonal preferred orientation may differentiate T-junctions. Walker et al. (1999) suggested that nonuniform surround suppression around CRFs may be useful for representing angles. Similar mechanisms may exist in area V1 of the monkey (Jones et al., 2001). As exemplified by cells 1 and 2 (Figs. 2, 3), we often observed that asymmetric direction profiles, which are unimodal or bimodal without a pair $180^{\circ}$ apart, are given by long half-lines but not by short half-lines. Although this tendency favors the contribution of the nonuniform surround suppression within area V2, additional study will be necessary to clarify the contribution of areas V1 and V2 to the formation of angle representation.

\section{Neuronal mechanisms for extracting angles in area V2}

Although we found that many V2 neurons are selective for angles, none specifically represented such features as angle width or angle orientation, regardless of the individual line components of angle stimuli. Instead, angle selectivity was closely related to the specific combination between the signals representing each line component. In one class of neurons, angle selectivity can be explained by an appropriate pattern of convergence of neural signals representing the individual line components of the angle stimulus, which may be accomplished by forward connection from area V1 or by lateral interactions within area V2. It is well accepted that at early visual stages neurons are tuned to a single optimal orientation, although some neurons reportedly show two optimal orientations in primate area V2 (Anzai and Van Essen, 2001, 2002) and in cat area 17 (Shevelev et al., 1994). Indeed, we observed that several neurons had bimodal or trimodal selectivity to half-line components not $180^{\circ}$ apart, and their peaks were often consistent with the direction of the two line components of the optimal angle. We also found, however, that the presence of either line component alone was not sufficient to elicit a response. This is clearly demonstrated by the fact that for wide and sharp angles, no neuron exhibited two axes of elonga- tion representing the two half-line components of the optimal angles. There thus appears to be another factor enabling fine selectivity, perhaps facilitatory interactions between the two preferred line components or suppression to nonpreferred line components.

In another class of neurons, such consistency was held only for one line component of the optimal angle. One candidate representing another line component is subthreshold excitatory interactions mediated by the long-range horizontal connections within area V2. Horizontal connections in the primary visual cortex are known to connect multiple cortical columns sharing the same orientation preference (Ts'o et al., 1986; Ts'o and Gilbert, 1988; Gilbert and Wiesel, 1989), but some V2 neurons having an orthogonal orientation preference show cross-correlation peaks near $0 \mathrm{msec}$, suggesting that horizontal connections may contribute to the formation of angle selectivity (Tamura et al., 1996). Complex integration beyond the CRFs has been observed in area V2 (von der Heydt and Peterhans, 1989; Bakin et al., 2000; Zhou et al., 2000). Another potential mechanism is inhibitory interactions with nonpreferred line components mediated by the nonuniform surround suppression. For instance, Heider et al. (2000) reported that some single end-stopped neurons showed selective responses to right corners. To explain the wide variety of angle selectivity observed in area V2, we also need to consider more complicated nonuniform surround suppression, as reported in other visual areas (Xiao et al., 1995; Walker et al., 1999; Jones et al., 2001).

\section{Angle representation in area $\mathrm{V} 2$ and beyond}

Fundamental to our understanding of the neuronal basis of object recognition is the idea that attribute-specific and locationspecific information are gradually integrated into a single object representation along the hierarchy of visual pathways. The present results are in line with the idea that the representation of complex stimulus features begins to emerge in area V2 (Kobatake and Tanaka, 1994; Hegde and Van Essen, 2000; Mahon and De Valois, 2001). In area V4, the next stage along the ventral visual pathway after area V2, a larger number of neurons are selective for complex stimulus features, including angles (Gallant et al., 1993, 1996; Kobatake and Tanaka, 1994; Pasupathy and Connor, 1999, 2001, 2002; Hanazawa and Komatsu, 2001). Their selectivity is invariant across several locations within CRFs. Furthermore, some neurons are selective for the angle orientation regardless of the direction of individual line components (Pasupathy and Connor, 1999). In contrast, the responses of angle-selective V2 neurons are related to signals representing each line component of the angle stimuli. The relatively small CRFs of V2 neurons did not allow us to examine the positional invariance within the CRFs, but it seems likely that a small positional shift would have a large effect on the responses and that these neurons would not have positional invariance. Thus, there seems to be a large difference in the angle representation in areas V2 and V4. We believe that signals extracted in area V2 are integrated in area V4 to more elaborately represent angles and curvatures. These differences in the nature of angle selectivity also suggest that feedback connections from area V4 are not likely the main source of angle selectivity observed in area V2.

\section{References}

Allman J, Miezin F, McGuinnes E (1985) Stimulus specific responses from beyond the classical receptive field: neurophysiological mechanisms for local-global comparisons in visual neurons. Annu Rev Neurosci 8:407430 . 
Anzai A, Van Essen DC (2001) Receptive field substructure of monkey V2 neurons in the orientation domain. Soc Neurosci Abstr 27:286.5.

Anzai A, Van Essen DC (2002) Receptive field structure of orientation selective cells in monkey V2. Soc Neurosci Abstr 28:720.12.

Bakin JS, Nakayama K, Gilbert CD (2000) Visual responses in monkey areas $\mathrm{V} 1$ and V2 to three-dimensional surface configurations. J Neurosci 20:8188-8198.

Barlow HB, Blakemore C, Pettigrew JD (1967) The neural mechanisms of binocular depth discrimination. J Physiol (Lond) 193:327-342.

Chen S, Levi DM (1996) Angle judgment: is the whole the sum of its parts? Vision Res 36:1721-1735.

Das A, Gilbert CD (1999) Topography of contextual modulations mediated by short-range interactions in primary visual cortex. Nature 399:655-661.

Desimone R, Albright TD, Gross CG, Bruce C (1984) Stimulus-selective properties of inferior temporal neurons in the macaque. J Neurosci 4:2051-2062.

Dobbins A, Zucker SW, Cynader MS (1987) Endstopped neurons in the visual cortex as a substrate for calculating curvature. Nature 329:438-441.

Dobbins A, Zucker SW, Cynader MS (1989) Endstopping and curvature. Vision Res 29:1371-1387.

Gallant JL, Braun J, Van Essen DC (1993) Selectivity for polar, hyperbolic, and Cartesian gratings in macaque visual cortex. Science 259:100-103.

Gallant JL, Connor CE, Rakshit S, Lewis JW, Van Essen DC (1996) Neural responses to polar, hyperbolic, and Cartesian gratings in area $\mathrm{V} 4$ of the macaque monkey. J Neurophysiol 76:2718-2739.

Gilbert CD, Wiesel TN (1989) Columnar specificity of intrinsic horizontal and corticocortical connections in cat visual cortex. J Neurosci 9:2432-2442.

Gilbert CD, Ito M, Kapadia M, Westheimer G (2000) Interactions between attention, context and learning in primary visual cortex. Vision Res 40:1217-1226.

Gross CG (1973) Visual functions of inferotemporal cortex. In: Handbook of sensory physiology (Jung R, ed), pp 451-482. Berlin: Springer.

Hammond P, Andrews DP (1978) Collinearity tolerance of cells in areas 17 and 18 of the cat's visual cortex: relative sensitivity to straight lines and chevrons. Exp Brain Res 31:329-339.

Hanazawa A, Komatsu H (2001) (2001) Influence of the direction of elemental luminance gradients on the responses of V4 cells to textured surfaces. J Neurosci 21:4490-4497.

Heeley DW, Buchanan-Smith HM (1996) Mechanisms specialized for the perception of image geometry. Vision Res 36:3607-3627.

Hegde J, Van Essen DC (2000) Selectivity for complex shapes in primate visual area V2. J Neurosci 20:RC 61(1-6).

Heggelund P, Hohmann A (1975) Responses of striate cortical cells to moving edges of different curvatures. Exp Brain Res 23:211-216.

Heider B, Meskenaite V, Peterhans E (2000) Anatomy and physiology of a neural mechanism defining depth order and contrast polarity at illusory contours. Eur J Neurosci 12:4117-4130.

Hubel DH, Wiesel TN (1959) Receptive fields of single neurons in the cat's striate cortex. J Physiol (Lond) 148:574-591.

Hubel DH, Wiesel TN (1965) Receptive fields and functional architecture in two nonstriate visual areas (18 and 19) of the cat. J Neurophysiol 28:229-289.

Hubel DH, Wiesel TN (1968) Receptive fields and functional architecture of monkey striate cortex. J Physiol (Lond) 195:215-243.

Jones HE, Grieve KL, Wang W, Sillito AM (2001) Surround suppression in primate V1. J Neurophysiol 86:2011-2028.
Judge SJ, Richmond BJ, Chu FC (1980) Implantation of magnetic search coils for measurement of eye position: an improved method. Vision Res 20:535-538.

Kobatake E, Tanaka K (1994) Neuronal selectivities to complex object features in the ventral visual pathway of the macaque cerebral cortex. J Neurophysiol 71:856-867.

Mahon LE, De Valois RL (2001) Cartesian and non-Cartesian responses in LGN, V1, and V2 cells. Vis Neurosci 18:973-981.

Pasupathy A, Connor CE (1999) Responses to contour features in macaque area V4. J Neurophysiol 82:2490-2502.

Pasupathy A, Connor CE (2001) Shape representation in area V4: positionspecific tuning for boundary conformation. J Neurophysiol 86:2505-2519.

Pasupathy A, Connor CE (2002) Population coding of shape in area V4. Nat Neurosci 5:1332-1338.

Regan D, Gray R, Hamstra SJ (1996) Evidence for a neural mechanism that encodes angles. Vision Res 36:323-330.

Shevelev IA, Lazareva NA, Novikova RV, Tikhomirov AS, Sharaev GA (1994) Double orientation tuning in the cat visual cortex units. Neuroscience 61:965-973.

Shevelev IA, Lazareva NA, Sharaev GA, Novikova RV, Tikhomirov AS (1998) Selective and invariant sensitivity to crosses and corners in cat striate neurons. Neuroscience 84:713-721.

Sillito AM, Grieve KL, Jones HE, Cudeiro J, Davis J (1995) Visual cortical mechanisms detecting focal orientation discontinuities. Nature 378:492-496.

Snodderly DM, Gur M (1995) Organization of striate cortex of alert, trained monkeys (Macaque fascicularis): ongoing activity, stimulus selectivity, and width of receptive field activating regions. J Neurophysiol $74: 2100-2125$.

Tamura H, Sato H, Katsuyama N, Hata Y, Tsumoto T (1996) Less segregated processing of visual information in $\mathrm{V} 2$ than in $\mathrm{V} 1$ of the monkey visual cortex. Eur J Neurosci 8:300-309.

Tanaka K (1993) Neuronal mechanisms of object recognition. Science 262: 685-688.

Tanaka K (1996) Inferotemporal cortex and object vision. Annu Rev Neurosci 19:109-139.

Ts'o DY, Gilbert CD (1988) The organization of chromatic and spatial interactions in the primate striate cortex. J Neurosci 8:1712-1727.

Ts'o DY, Gilbert CD, Wiesel TN (1986) Relationships between horizontal interactions and functional architecture in cat striate cortex as revealed by cross-correlation analysis. J Neurosci 6:1160-1170.

Versavel M, Orban GA, Lagae L (1990) Responses of visual cortical neurons to curved stimuli and chevrons. Vision Res 30:235-248.

von der Heydt R, Peterhans E (1989) Mechanisms of contour perception in monkey visual cortex. 1. Lines of pattern discontinuity. J Neurosci 9:1731-1748.

Walker GA, Ohzawa I, Freeman RD (1999) Asymmetric suppression outside the classical receptive field of the visual cortex. J Neurosci 19:10536-10553.

Wolbarsht ML, MacNichol EF, Wagner HG (1960) Glass insulated platinum microelectrode. Science 132:1309-1310.

Xiao D-K, Raiguel S, Marcar V, Koenderink J, Orban GA (1995) Spatial heterogeneity of inhibitory surrounds in the middle temporal visual area. Proc Natl Acad Sci USA 92:11303-11306.

Zhou H, Friedman HS, von der Heydt R (2000) Coding of border ownership in monkey visual cortex. J Neurosci 20:6594-6611. 